\title{
Economic Burden of Irritable Bowel Syndrome with Diarrhea: Retrospective Analysis of a U.S. Commercially Insured Population
}

\author{
Jessica L. Buono, MPH; Kush Mathur, MS; Amelia J. Averitt, MPH; and David A. Andrae, PhD
}

\begin{abstract}
BACKGROUND: The economic burden associated with irritable bowel syndrome with diarrhea (IBS-D) is not well understood.

OBJECTIVES: To (a) evaluate total annual all-cause, gastrointestinal (GI)related, and symptom-related (i.e., IBS, diarrhea, abdominal pain) health care resource use and costs among IBS-D patients in a U.S. commercially insured population and (b) estimate incremental all-cause health care costs of IBS-D patients versus matched controls.
\end{abstract}

METHODS: Patients aged $\geq 18$ years with 12 months of continuous medical and pharmacy benefit eligibility in $\mathbf{2 0 1 3}$ were identified from the Truven Health MarketScan research database. The study sample included patients with $\geq 1$ medical claim with an International Classification of Diseases, Ninth Revision, Clinical Modification (ICD-9-CM) diagnosis code in any position for IBS (ICD-9-CM 564.1x) and either (a) $\geq 2$ claims for diarrhea (ICD-9-CM $787.91,564.5 x)$ on different service dates in 2013 , or (b) $\geq 1$ claim for diarrhea plus $\geq 1$ claim for abdominal pain (ICD-9-CM 789.0x) on different service dates in 2013 , or (c) $\geq 1$ claim for diarrhea plus $\geq 1$ pharmacy claim for a symptom-related prescription on different service dates in 2013. Controls included patients with no claims for IBS, diarrhea, abdominal pain, or symptom-related prescriptions in 2013. Controls were randomly selected and matched with IBS-D patients in a 1:1 ratio based on age ( \pm 4 years), gender, geographic location, and health plan type. All-cause health care resource utilization included medical and pharmacy claims for health care services associated with any condition. Total health care costs were defined as the sum of health plan-paid and patient-paid direct health care costs from prescriptions and medical services, including inpatient, emergency department (ED), and physician office visits, and other outpatient services. A total cost approach was used to assess all-cause, Gl-related, and symptom-related health care costs for IBS-D patients. An incremental cost approach via generalized linear models was used to assess the excess all-cause costs attributable to IBS-D after adjusting for demographics and general and GI comorbidities.

RESULTS: Of 39,306 patients ( $n=19,653$ each for IBS-D and matched controls) included, mean $( \pm S D)$ age was $47( \pm 17)$ years and $76.5 \%$ were female. Compared with controls, IBS-D patients had a significantly higher mean annual number of hospitalizations, ED visits, office visits, and monthly (30-day) prescription fills. Mean annual all-cause health care costs for IBS-D patients were $\$ 13,038$, with over half (58.4\%) attributable to office visits and other outpatient services (e.g., diagnostic tests and laboratory or radiology services), and remaining costs attributable to prescriptions (19.5\%), inpatient admissions (13.6\%), and ED visits (8.5\%). GI-related $(\$ 3,817)$ and symptomrelated $(\$ 1,693)$ costs were also primarily driven by other outpatient service costs. After adjusting for demographics and comorbidities, incremental annual all-cause costs associated with IBS-D were $\$ 2,268$ ( $\$ 9,436$ for IBS-D patients vs. $\$ 7,169$ for matched controls; $P<0.001$ ) per patient/year, of which $78 \%$ were from medical costs and $22 \%$ were from prescription costs.

CONCLUSIONS: IBS-D was associated with a substantial burden in direct costs in this population. Compared with matched controls, IBS-D patients had greater medical service use and incurred significantly more annual all-cause health care costs, even after controlling for demographics and comorbidities.
Incremental costs associated with IBS-D were primarily attributable to increased use of medical services rather than pharmacy costs.

J Manag Care Spec Pharm. 2017;23(4):453-60

Copyright $\odot 2017$, Academy of Managed Care Pharmacy. All rights reserved.

\section{What is already known about this subject}

Previous studies have demonstrated a significant economic burden associated with irritable bowel syndrome (IBS) overall and among patients with IBS with constipation (IBS-C); however, data specific to the economic burden of IBS with diarrhea (IBS-D) are lacking. The total cost difference for patients with IBS versus matched controls has been estimated at $\$ 2,026$ among patients in a health maintenance organization and $\$ 4,120$ for patients with IBS-C in a U.S. commercially insured population.

\section{What this study adds}

This study evaluated the economic burden associated with IBS-D among patients in a commercially insured population using 2 cost of illness approaches - the total cost approach and the incremental cost approach-to provide a rigorous assessment of the cost of illness of IBS-D.

Total annual all-cause costs were significantly higher among IBS-D patients compared with matched controls, with an incremental difference of $\$ 2,268$ per patient per year after adjustment for demographic characteristics and comorbidities.

Incremental annual all-cause costs associated with IBS-D were primarily driven by costs associated with increased use of medical services, which accounted for $78 \%$ of incremental all-cause costs; these costs were largely attributable to outpatient service use.

T rritable bowel syndrome (IBS) is a chronic functional gastrointestinal (GI) disorder characterized by abdominal 1 pain or discomfort and altered bowel habits in the absence of demonstrable organic disease. ${ }^{1,2}$ IBS is the condition most commonly diagnosed by gastroenterologists, accounts for $12 \%$ of diagnoses in primary care practices, and is one of the top 10 reasons patients consult a primary care physician. ${ }^{3}$ The Rome III diagnostic criteria ${ }^{2}$ represent the current gold standard for the diagnosis of IBS and include symptoms of recurrent abdominal pain or discomfort for $\geq 3$ days per month over the past 3 months that are associated with $\geq 2$ of the following: 
improvement with defecation; onset associated with a change in stool frequency; or onset associated with a change in stool form. IBS is classified into subtypes based on the predominant stool pattern, including IBS with constipation (IBS-C), IBS with diarrhea (IBS-D), and IBS with mixed bowel patterns of both constipation and diarrhea (IBS-M). ${ }^{2}$ IBS-D is defined as loose, mushy, or watery stools for $\geq 25 \%$ of bowel movements and hard or lumpy stools for $<25 \%$ of bowel movements in the absence of antidiarrheal or laxative use. ${ }^{2}$

IBS affects approximately $10 \%$ to $15 \%$ of adults in the United States, with IBS-D estimated to account for approximately one third of cases. ${ }^{4}$ Due to the disruptiveness of symptoms, IBS patients seek health care to diagnose and manage their condition, resulting in increased health care resource utilization and a substantial economic burden on the health care system., ${ }^{5,6}$ While there exists a paucity of data on the burden specifically related to the IBS-D subtype, IBS in general represents a significant economic burden in terms of both direct costs (including increased health care resource utilization) and indirect costs associated with lost work productivity. ${ }^{3}$ Total direct costs associated with IBS have been estimated at $\$ 6,182$ per IBS patient per year compared with $\$ 4,156$ for age- and sex-matched controls (2013 U.S. dollars [USD] [inflation-adjusted 2001 USD]) among patients in a U.S. health maintenance organization. ${ }^{7}$ Among patients with IBS-C, the incremental annual all-cause costs have been estimated at $\$ 4,120$ (2013 USD [inflationadjusted 2010 USD]) per patient per year (after adjustment for demographics and comorbidities), the majority of which were attributable to medical services. ${ }^{8}$

As IBS subtypes differ in terms of their symptomatology and appropriate management strategies, ${ }^{9-11}$ this may result in differences in the associated economic burden between IBS overall and the individual subtypes. Thus, the purpose of this study was to examine the annual health care resource utilization and direct costs associated with IBS-D by summarizing the total annual all-cause, GI-related, and symptom-related health care resource use and costs among patients with IBS-D and estimating the total annual incremental all-cause health care costs among patients with IBS-D relative to matched controls.

\section{Methods}

\section{Data Source}

Medical, pharmacy, and eligibility claims data were extracted from the Truven Health MarketScan research database, which includes annual data on over 50 million covered lives, reflecting a nationally representative data sample of the U.S. population. The database consists of inpatient and outpatient medical and surgical claims data and outpatient pharmacy claims data from active employees, early retirees, COBRA continuees, and dependents insured by employer-sponsored plans.

All data used in this study were de-identified and accessed with protocols compliant with the Health Insurance Portability and Accountability Act of 1996 regulations. Patient confidentiality was preserved, and the anonymity of all patient data was safeguarded throughout the study. No waiver of informed consent was required from an institutional review board.

\section{Study Design}

This study was a retrospective administrative claims database analysis of beneficiaries in a U.S. commercially insured population. To assess all-cause, GI-related, and symptom-related health care resource utilization and costs associated with IBS-D, all-cause and GI-related outcomes were compared between patients with IBS-D and their matched controls. Controls were randomly selected and matched with patients with IBS-D in a 1:1 ratio based on age ( \pm 4 years), gender, geographic location (state), and health plan type. Matching on geographic location and health plan type was undertaken to control for differences in state practice patterns and health plans that might confound estimates of the incremental health care costs of patients with IBS-D versus those without IBS-D.

Total costs were assessed as the sum of all-cause, GI-related, and symptom-related health care costs for patients with IBS-D, and incremental costs were estimated as the excess all-cause costs attributable solely to the presence of IBS-D. Due to the chronic nature of IBS-D, with patients experiencing symptoms that may wax and wane over long periods of time, a prevalence-based approach was employed to estimate the economic burden of illness, ${ }^{12}$ and a 1-year claims-eligibility window was used to assess the annual costs of IBS-D. The study period was defined as January 1 through December 31, 2013, based on the most recent calendar-year data available at the time this study was conducted.

\section{Study Population}

The study population included patients aged $\geq 18$ years with 12 months of continuous medical and pharmacy benefit eligibility in 2013. Given the lack of an International Classification of Diseases, Ninth Revision, Clinical Modification (ICD-9-CM) diagnosis code for IBS-D, inclusion and exclusion criteria used to define the study sample were developed based on prior administrative claims research in IBS- $\mathrm{C}^{8}$ and in consultation with gastroenterologists on the most appropriate criteria for defining IBS-D patients based on claims. Patients were classified as having IBS-D if they had $\geq 1$ medical claim with an ICD-9-CM diagnosis code in any position for IBS (ICD-9-CM 564.1x) in 2013 and either (a) $\geq 2$ medical claims for diarrhea (ICD-9-CM 787.91, 564.5x) on different dates of service in 2013 , or (b) $\geq 1$ medical claim for diarrhea plus $\geq 1$ medical claim for abdominal pain (ICD-9-CM 789.0x) on different dates of service in 2013, or (c) $\geq 1$ medical claim for diarrhea plus $\geq 1$ pharmacy claim for a symptom-related (i.e., IBS, diarrhea, and abdominal pain) prescription, including alosetron, EnteraGam, probiotics, antidiarrheals, tricyclic antidepressants/selective 
serotonin reuptake inhibitors (only if no concomitant diagnosis of depression), bulk-forming agents, anticholinergics/antispasmodics, and rifaximin (only if no concomitant diagnosis of traveler's diarrhea or hepatic encephalopathy) on different dates of service in 2013.

To avoid inclusion of patients with IBS-C, IBS-M, chronic constipation, drug-induced diarrhea, or other GI conditions or medications that may affect GI system function, patients were excluded if they met any of the following criteria: (a) $\geq 2$ medical claims for constipation, chronic gastritis, chronic pancreatitis, chronic duodenitis, or uterine fibroids occurring at least 30 days apart during the study period or $(b) \geq 1$ medical claim for constipation plus $\geq 1$ pharmacy claim for a constipation-related prescription (including linaclotide, lubiprostone, stimulant laxatives, osmotic laxatives, bulk-forming agents, or stool softeners) occurring at least 30 days apart during the study period. Patients were also excluded if they had any medical claim for conditions that may be misdiagnosed as IBS-D or present with similar symptomatology (i.e., GI malignancy, Crohn's disease, ulcerative colitis, celiac disease, vascular insufficiency of intestine, intestinal malabsorption, diverticulitis, diabetic neuropathy, HIV, radiation- or chemotherapy-induced colitis, microscopic colitis [lymphocytic or collagenous colitis], carcinoid syndrome, Zollinger-Ellison syndrome, uncontrolled hyperthyroidism, laxative abuse, diarrhea that is clearly drug induced, lactose intolerance, short-bowel syndrome, dumping syndrome, intestinal bypass surgery, gastric bypass/gastrectomy, collagen vascular diseases, eosinophilic gastroenteritis, cystic fibrosis, or small intestinal bacterial overgrowth).

Exclusion criteria applied in the identification of IBS-D patients were also applied in defining the study sample of matched controls. In addition, controls could not have any medical claims for IBS, diarrhea, or abdominal pain or pharmacy claims for symptom-related prescriptions during the study period.

\section{Study Measures}

Study measures included all-cause, GI-related, and symptomrelated (i.e., IBS, diarrhea, and abdominal pain) health care resource utilization and costs. All-cause health care resource utilization was defined as all medical (inpatient admissions, emergency department [ED] visits, physician office visits, and other outpatient services, including diagnostic tests and laboratory or radiology services) and pharmacy (prescription drug use) claims for health care services associated with any condition. All-cause prescription use was assessed as the mean number of standardized monthly (30-day) all-cause prescription fills calculated by dividing the annual days supply of each prescription by 30 days.

Total all-cause health care costs were defined as the sum of health plan-paid and patient-paid all-cause direct health care costs incurred from all medical and pharmacy claims associated with any condition during the study period. GI-related health care resource utilization and costs were defined as resource use and direct costs attributable to medical and pharmacy claims associated with any of the following GI-related conditions, selected based on clinically relevant literature and expert clinical opinion: gastroesophageal reflux disease, esophagitis, dyspepsia, gallbladder or biliary disease, GI hemorrhage, hemorrhoids, nausea, vomiting, gastroenteritis, pancreatitis, ischemic colitis, IBS, diarrhea, and abdominal pain..$^{8,13-15}$ Symptomrelated health care resource utilization and costs were defined as health care services and costs associated with medical and pharmacy claims for IBS, diarrhea, and abdominal pain as the primary diagnosis during the study period.

Patient comorbidities were captured using the Elixhauser Comorbidity Index (ECI), a claims-based measure of overall disease burden based on the occurrence of at least 1 of more than 31 comorbid conditions identified using the ICD-9-CM coding manual. ${ }^{16}$ In addition to the ECI comorbidities, 14 general and 11 GI-related comorbidities identified based on clinically relevant literature and expert clinical opinion as being common comorbid conditions or comorbid consequences of IBS-D were also assessed..$^{13-15}$

\section{Statistical Analyses}

Descriptive analyses were employed to investigate the frequency and significance of characteristics between IBS-D patients and matched controls, while multivariable analyses were used to assess the all-cause incremental costs of IBS-D. For continuous variables (e.g., age), means, standard deviations (SDs), and medians were reported; frequencies and percentages were reported for categorical variables (e.g., gender). To assess incremental costs, data were adjusted for demographic characteristics and comorbidities using a generalized linear model with the recycled predictions approach and presented as both adjusted and unadjusted excess costs. For descriptive analyses, IBS-D patients were compared with controls matched on demographic characteristics. To compare all-cause health care resource utilization and costs between patients with IBS-D and matched controls, McNemar's tests for matched pairs were used for categorical variables and nonparametric bootstrap estimation and t-tests were used for continuous variables. Nonparametric bootstrap estimation was performed with 3,000 replicates with replacement and a sample size of 1,000 generated by randomly drawing samples from the total population clustered by the matched identifier.

Generalized linear models were used to assess the incremental all-cause costs attributable to IBS-D after adjusting for demographics, ECI score, and 14 general and 11 GI-related comorbidities not included in the ECI score (see Table 1 for a list of general and GI-related comorbidities controlled for in these analyses). A recycled predictions method was used in conjunction with the generalized linear models to calculate the marginal 


\begin{tabular}{|c|c|c|c|}
\hline Variable & $\begin{array}{c}\text { Patients with } \\
\text { IBS-D } \\
(\mathrm{n}=19,653)\end{array}$ & $\begin{array}{c}\text { Matched } \\
\text { Controls } \\
(\mathrm{n}=19,653) \\
\end{array}$ & $P$ Value \\
\hline Age, mean \pm SD & $47.1 \pm 17.2$ & $47.1 \pm 17.2$ & $>0.999$ \\
\hline Female, n (\%) & $15,033(76.5)$ & $15,033(76.5)$ & $>0.999$ \\
\hline \multicolumn{4}{|l|}{ Health plan type, $\mathrm{n}(\%)$} \\
\hline $\mathrm{PPO}$ & $11,947(60.8)$ & $11,947(60.8)$ & $>0.999$ \\
\hline $\mathrm{HMO}$ & $2,834(14.4)$ & $2,834(14.4)$ & $>0.999$ \\
\hline Other commercial plan type ${ }^{b}$ & $4,872(24.8)$ & $4,872(24.8)$ & $>0.999$ \\
\hline \multicolumn{4}{|l|}{ Geographic region, $\mathrm{n}(\%)$} \\
\hline Northeast & $4,056(20.6)$ & $4,056(20.6)$ & $>0.999$ \\
\hline North Central & $4,864(24.7)$ & $4,864(24.7)$ & $>0.999$ \\
\hline South & $6,735(34.3)$ & $6,735(34.3)$ & $>0.999$ \\
\hline West & $3,998(20.3)$ & $3,998(20.3)$ & $>0.999$ \\
\hline ECI score, mean \pm SD & $1.8 \pm 1.9$ & $0.8 \pm 1.3$ & $<0.001$ \\
\hline \multicolumn{4}{|l|}{ General comorbidities, n (\%) } \\
\hline Functional/chronic pain disorders & $7,136(36.3)$ & $3,300(16.8)$ & $<0.001$ \\
\hline Hypertension & $6,356(32.3)$ & $4,338(22.3)$ & $<0.001$ \\
\hline Hyperlipidemia & $5,257(26.7)$ & $3,408(17.3)$ & $<0.001$ \\
\hline Anxiety & $4,802(24.4)$ & $803(4.1)$ & $<0.001$ \\
\hline Malaise and fatigue & $4,658(23.7)$ & $1,348(6.9)$ & $<0.001$ \\
\hline Depression & $4,553(23.2)$ & $924(4.7)$ & $<0.001$ \\
\hline Urinary tract infection & $3,002(15.3)$ & $1,041 \quad(5.2)$ & $<0.001$ \\
\hline Headache & $2,604(13.2)$ & $683(3.5)$ & $<0.001$ \\
\hline Upper respiratory infection & $2,508(12.8)$ & $1,031 \quad(5.2)$ & $<0.001$ \\
\hline Myalgia and myositis & $1,981(10.1)$ & $476(2.4)$ & $<0.001$ \\
\hline Fibromyalgia & $1,981(10.1)$ & $476(2.4)$ & $<0.001$ \\
\hline Dizziness & $1,660 \quad(8.4)$ & $498(2.5)$ & $<0.001$ \\
\hline Insomnia & $1,349(6.9)$ & $278(1.4)$ & $<0.001$ \\
\hline Dysuria & $1,305(6.6)$ & $373(1.9)$ & $<0.001$ \\
\hline \multicolumn{4}{|l|}{ GI-related comorbidities, n (\%) } \\
\hline GERD & $6,243(31.8)$ & $858 \quad(4.4)$ & $<0.001$ \\
\hline Hemorrhoids & $3,075(15.6)$ & $362(1.8)$ & $<0.001$ \\
\hline Nausea & $2,764(14.1)$ & $99(0.5)$ & $<0.001$ \\
\hline Esophagitis & $2,072(10.5)$ & $186(0.9)$ & $<0.001$ \\
\hline GI hemorrhage & $1,461 \quad(7.4)$ & $76(0.4)$ & $<0.001$ \\
\hline Dyspepsia & $1,430 \quad(7.3)$ & $53(0.3)$ & $<0.001$ \\
\hline Vomiting & $914(4.7)$ & $33(0.2)$ & $<0.001$ \\
\hline Gallbladder or biliary disease & $645(3.3)$ & $47 \quad(0.2)$ & $<0.001$ \\
\hline Pancreatitis & $198(1.0)$ & $5(0.03)$ & $<0.001$ \\
\hline Gastroenteritis & $46(0.2)$ & $3(0.02)$ & $<0.001$ \\
\hline Ischemic colitis & $0(0.0)$ & $0(0.0)$ & - \\
\hline \multicolumn{4}{|c|}{$\begin{array}{l}\text { aAs of January 1, } 2013 . \\
\text { bIncludes comprehensive noncapitated point-of-service, consumer-driven health plan, } \\
\text { high-deductible health plan, exclusive provider organization, and point-of service with } \\
\text { capitation (capitated or partially capitated) health plans. } \\
\text { ECI=Elixhauser Comorbidity Index; GERD=gastroesophageal reflux disease; } \\
\text { GI= gastrointestinal; HMO=health maintenance organization; IBS-D=irritable } \\
\text { bowel syndrome with diarrhea; } P P O=\text { preferred provider organization; } S D=\text { standard } \\
\text { deviation. }\end{array}$} \\
\hline
\end{tabular}

all-cause costs for both IBS-D patients and matched controls. Nonparametric bootstrap estimation and t-tests were used to further assess the significance of any differences in adjusted

\section{FIGURE 1 Patient Identification Flow Chart}

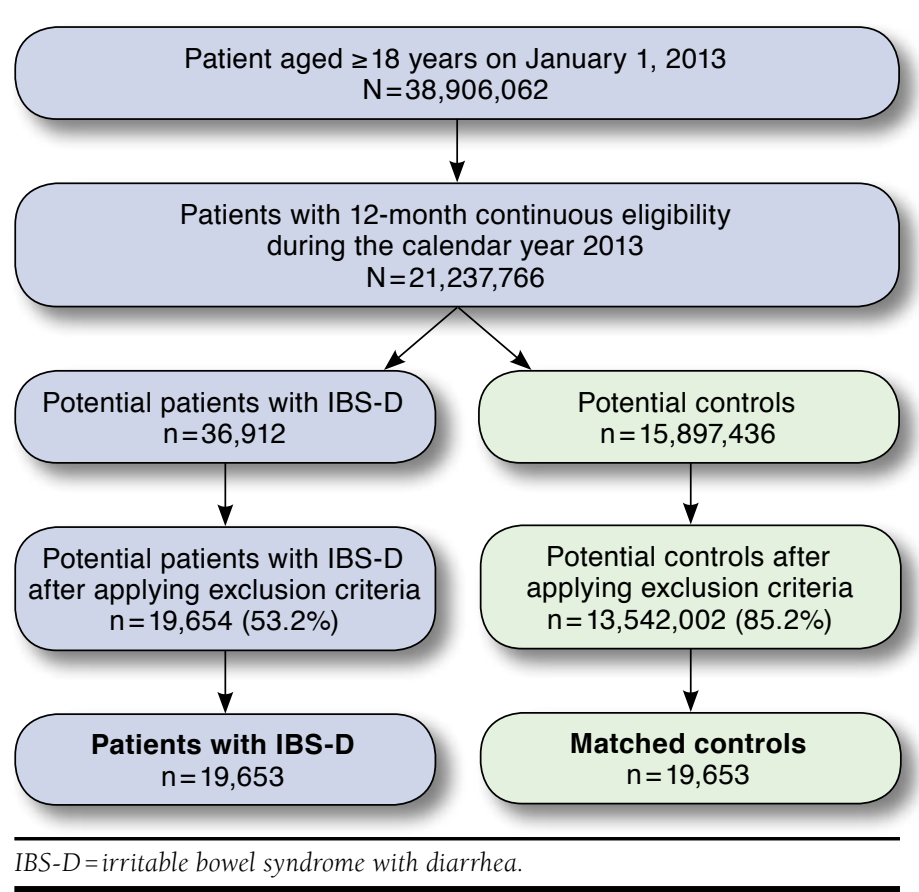

incremental all-cause medical costs and incremental all-cause prescription costs. This methodology was used to avoid omission of covariates in the generalized linear models that might have had significant explanatory power or interaction.

Differences were considered significant at the $a<0.05$ level and all data analyses were conducted using SAS version 9.2 (SAS, Cary, NC).

\section{Results}

\section{Demographic and Clinical Characteristics}

A total of 39,306 patients ( $n=19,653$ each for IBS-D and matched controls) met the inclusion criteria and were included in the study (Figure 1). The mean $( \pm$ SD) age was $47( \pm 17)$ years, $76.5 \%$ of patients were female, and the majority of patients (60.8\%) had a preferred provider organization health care plan (Table 1). Patients with IBS-D had a significantly higher mean ECI score (1.8 vs. $0.8 ; P<0.001)$ and a significantly higher incidence of general and GI-related comorbidities compared with matched controls (Table 1).

\section{Total Annual Health Care Resource Utilization and Costs}

Compared with matched controls, patients with IBS-D had a significantly higher mean annual number of all-cause hospitalizations, ED visits, physician office visits, and monthly (30-day) prescription fills (Figure 2). In particular, patients with IBS-D had more than triple the mean (SD) annual number 


\section{FIGURE 2 Mean Annual All-Cause Health Care Resource Utilization Among Patients with} IBS-D Compared with Matched Controls

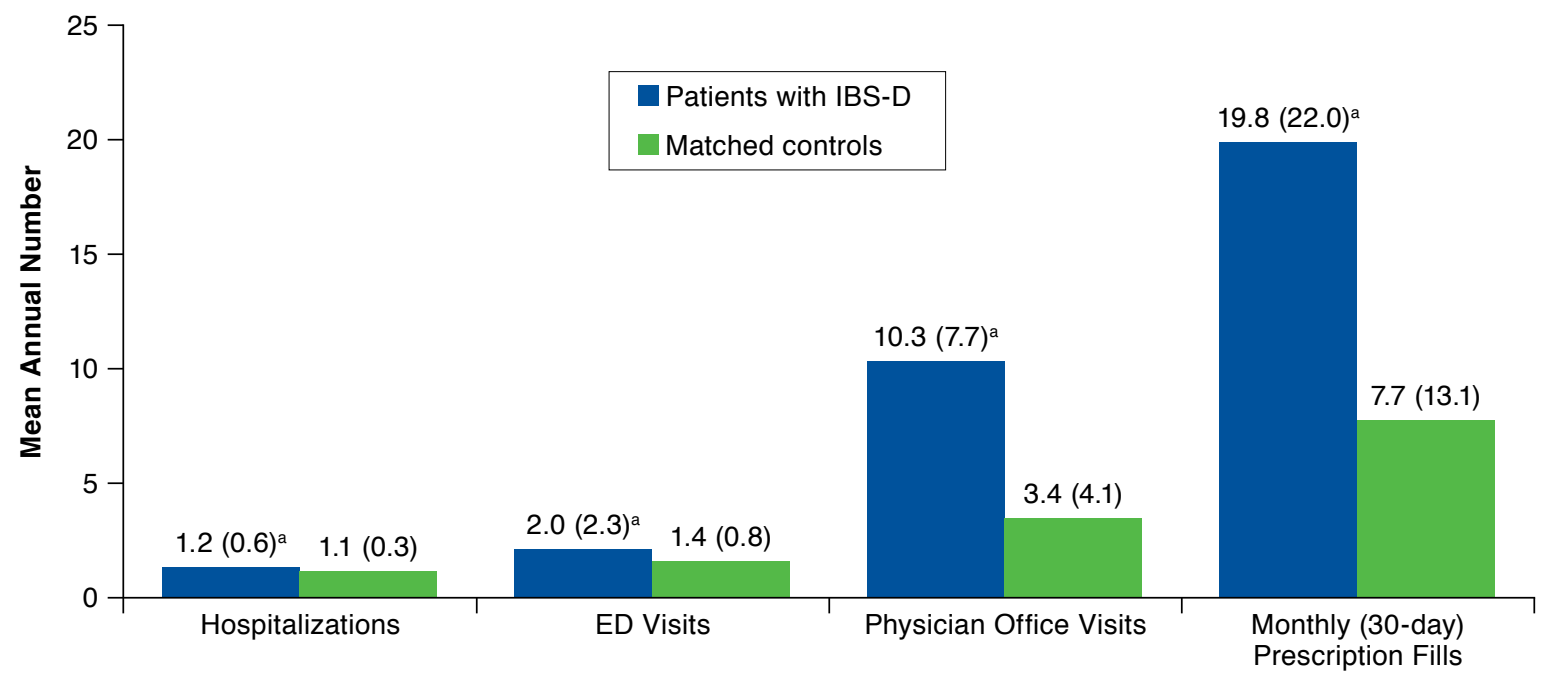

Note: Data are shown as mean (standard deviation).

aP $<0.001$.

$E D=$ emergency department; IBS-D = irritable bowel syndrome with diarrhea.

of physician office visits (10.3 [7.7] vs. 3.4 [4.1]; $P<0.001)$ and more than double the mean (SD) number of monthly (30-day) prescription fills (19.8 [22.0] vs. 7.7 [13.1]; $P<0.001)$ annually compared with matched controls.

Among IBS-D patients, mean (SD) annual all-cause health care costs were $\$ 13,038$ ( $\$ 24,645)$, half of which $(50.3 \%)$ were attributable to other outpatient services (diagnostic tests and laboratory or radiology services). Remaining all-cause costs were due to prescriptions (19.5\%), inpatient admissions (13.6\%), ED visits (8.5\%), and physician office visits (8.1\%) (Figure 3). Of the total all-cause costs, 29.3\% $(\$ 3,817)$ were attributable to GI-related costs and $13.0 \%(\$ 1,693)$ to symptom-related costs. The majority of GI- and symptom-related costs were also driven primarily by costs associated with other outpatient services ( $47.4 \%$ and $50.9 \%$, respectively). Notably, costs associated with endoscopic procedures accounted for $15.5 \%$ of symptom-related costs, with $23.8 \%$ of patients with IBS-D receiving $\geq 1$ endoscopic procedure.

\section{Incremental Costs of IBS-D}

Patients with IBS-D incurred $\$ 8,768$ more in unadjusted mean annual all-cause costs compared with matched controls $(P<0.001)$, with an incremental difference of $\$ 7,273(83 \%)$ from medical costs and $\$ 1,494$ (17\%) from prescription costs (Figure 4). After adjusting for demographic characteristics and comorbidities, the incremental total all-cause health care costs attributable to IBS-D were $\$ 2,268(P<0.001)$, of which $\$ 1,768$
(78\%) were from medical costs and $\$ 499$ (22\%) were from prescription costs (Figure 4).

\section{Discussion}

This study demonstrates a significant economic burden associated with IBS-D, even after controlling for demographic characteristics and general and GI-related comorbidities. To our knowledge, this is the first study to address direct annual health care costs specific to the IBS-D subtype. As IBS-D is a chronic condition in which patients are symptomatic for a protracted period, a prevalence-based approach was used to estimate the economic burden of illness, and both total and incremental costs were assessed, making these findings particularly relevant from a payer perspective. In addition, an exact matching method based on age, gender, geographic location, and health plan type was used to ensure that matched controls were as similar as possible to patients with IBS-D. This study also controlled for an extensive list of comorbid conditions in order to isolate the costs associated with IBS-D.

In this study population, patients identified as having IBS-D had significantly greater mean annual use of medical services compared with matched controls, including more than triple the number of physician office visits and more than double the number of monthly (30-day) prescription fills. Costs associated withoutpatientservices, suchaslaboratoryorradiology services and diagnostic tests, were the primary driver of annual all-cause, GI-related, and symptom-related costs among 


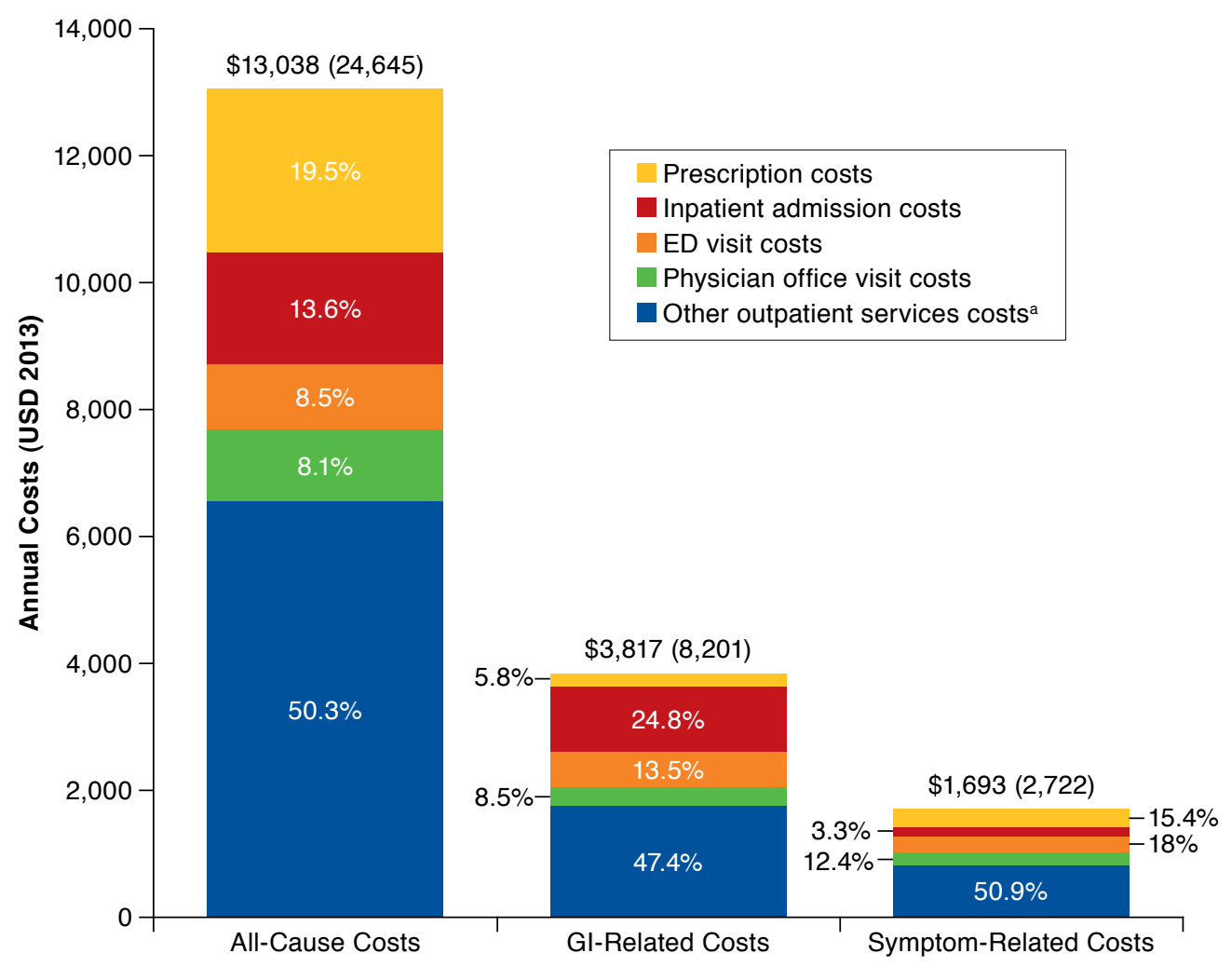

Note: Data are shown as mean (standard deviation).

aOther outpatient services costs include diagnostic tests and laboratory or radiology services.

$E D=$ emergency department; $G I=$ gastrointestinal; $I B S-D=$ irritable bowel syndrome with diarrhea; USD =U.S. dollars.

patients with IBS-D, contributing to around $50 \%$ of each of these costs. Patients with IBS-D incurred significantly higher adjusted all-cause costs compared with matched controls, with nearly $80 \%$ of incremental costs attributable to more frequent use of medical services and a smaller proportion resulting from prescription costs. These results suggest a potential opportunity for reducing health care resource utilization and costs associated with IBS-D with the use of effective therapies that provide adequate and sustained relief of the multiple symptoms of IBS-D.

The direct costs of IBS-D can be put into perspective by comparing them with the total annual health care costs for patients with other chronic conditions, such as asthma and migraine, for which symptoms also wax and wane over time. Total annual per patient costs of IBS-D found in this study are comparable to those attributable to asthma and migraine. ${ }^{17,18}$ Although such comparisons should be made with caution, given that methodologies differ across studies, the similarities between the costs associated with asthma and migraine and those associated with IBS-D are important benchmarks, since asthma and migraine tend to have greater recognition among payers regarding the associated costs to the health care system. The comparability of IBS-D with these other conditions in terms of cost burden represents an important finding for the understanding of the burden associated with IBS-D.

\section{Limitations}

As with any retrospective claims database analysis, the results of this study should be interpreted in light of certain limitations. First, definitive diagnoses are not available in claims data, and while ICD-10-CM includes a diagnosis code for IBS-D, no specific ICD-9-CM diagnosis code exists in the current claims data for IBS-D. The lack of a diagnosis code and the inconsistent use of ICD-9-CM diagnosis codes for IBS and diarrhea as standalone conditions impede the accurate identification of patients with IBS-D in current administrative claims database analyses. Accordingly, classification of patients as potential IBS-D cases was informed by expert clinical 
FIGURE 4 Unadjusted and Adjusted Mean Medical and Prescription Costs Among Patients with IBS-D and Matched Controls

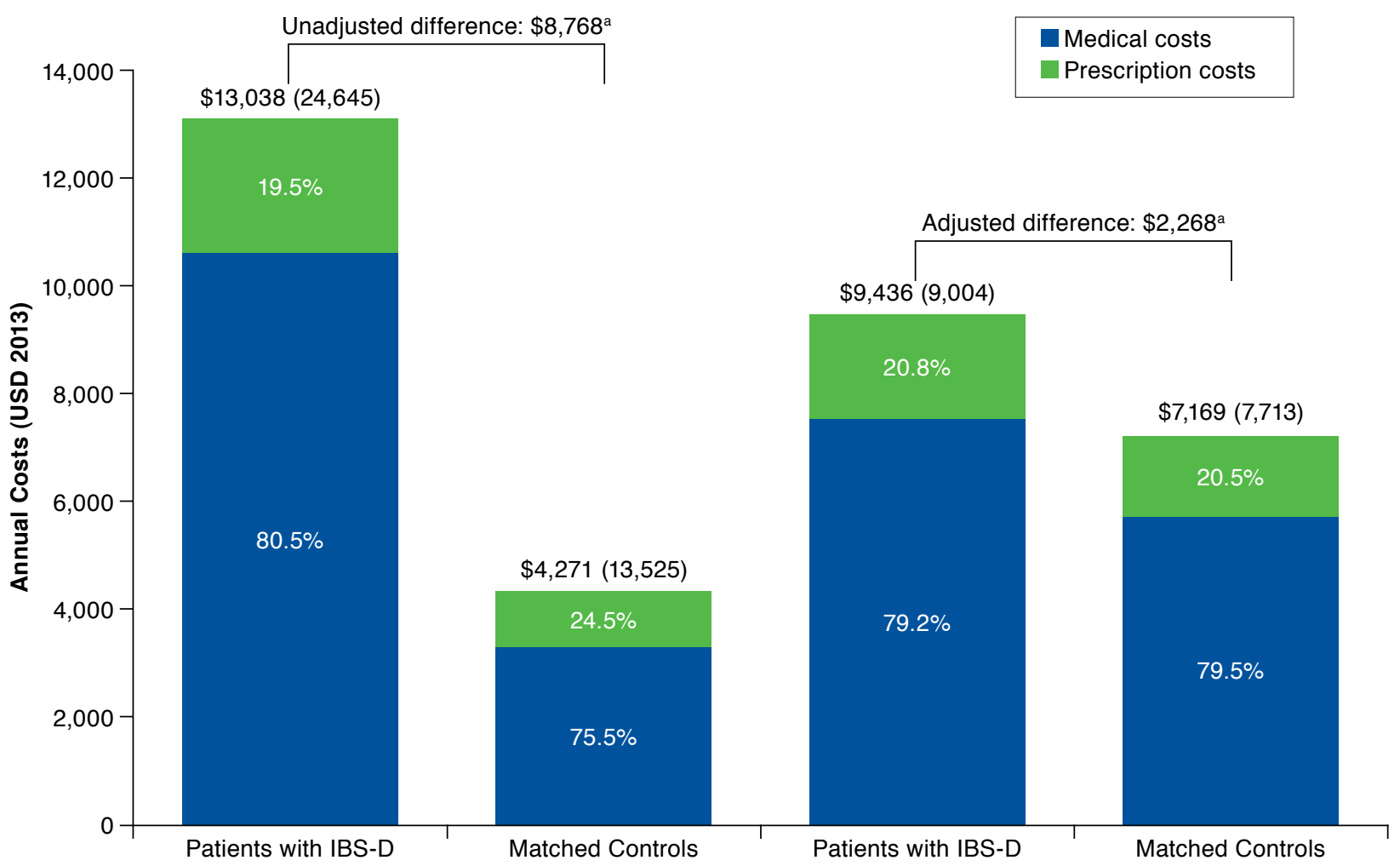

Note: Data are shown as mean (standard deviation).

aP $<0.001$.

IBS-D = irritable bowel syndrome with diarrhea; USD =U.S. dollars.

opinion and prior claims analyses in IBS-C, ${ }^{8}$ and identification criteria were constructed based on available diagnostic codes and pharmacy prescriptions, which may have led to potential classification bias affecting the cost estimates. However, misclassification of patients within a study would be expected to bias results in such a way that the groups appear more similar than if misclassification were minimal. To the extent that ICD9-CM codes must be used to examine resource use and costs among IBS-D patients, further research should be undertaken to validate the identification of these patients through a retrospective chart review to confirm diagnoses of IBS-D identified through claims until a specific diagnosis code for IBS-D is available in claims databases.

Second, while patients in this study were matched to controls based on age, gender, geographic location, and health plan, and analyses were controlled for ECI score and 25 other general and GI-related comorbidities, the incremental cost estimates may have been affected by residual confounding of unmeasured variables. Finally, this study only identified patients with IBS-D who were commercially insured, thereby limiting the generalizability of the findings to other populations, such as uninsured individuals or Medicare or Medicaid recipients. Future studies should assess patterns of health care resource utilization and costs associated with IBS-D in other patient populations and identify opportunities for more effective disease management to potentially reduce the costs associated with this disorder.

\section{Conclusions}

This study demonstrated significant direct costs and greater use of medical services among patients with IBS-D compared with matched controls. The majority of incremental costs were due to increased medical costs, and IBS-D-related costs were largely attributable to a greater use of physician office visits and other outpatient services, suggesting increased health careseeking behavior among patients with IBS-D and indicating a lack of effective therapies to treat the multiple symptoms of 
IBS-D. Further research should focus on the underlying factors driving increased medical resource use among patients with IBS-D and how effective treatments can potentially alleviate the excess medical costs associated with this condition.

\section{Authors}

JESSICA L. BUONO, MPH, and DAVID A. ANDRAE, PhD, Allergan, Jersey City, New Jersey. KUSH MATHUR, MS, and AMELIA J. AVERITT, MPH, Axtria, Berkeley Heights, New Jersey.

AUTHOR CORRESPONDENCE: Jessica L. Buono, MPH, 185 Hudson St., Jersey City, NJ 07931. Tel.: 201.427.8292; E-mail: Jessica.Abel@allergan.com.

\section{DISCLOSURES}

This study was funded by Allergan. The authors received no compensation related to the development of the manuscript. Buono and Andrae are employees of Allergan. Mathur is an employee of Axtria. Averitt was an employee of Axtria at the time this study was conducted.

Data from this manuscript have previously been presented in poster format by Buono at the American College of Gastroenterology Annual Scientific Meeting; Honolulu, Hawaii; October 16-21, 2015.

Mathur and Averitt were involved in conducting the study analyses. All authors were involved in the study design, interpretation of the data, and preparation of the manuscript. The authors take full responsibility for the scope, direction, and content of the manuscript and have approved the submitted manuscript.

\section{ACKNOWLEDGMENTS}

The authors thank Steven J. Shiff, MD, and Paul S. Covington, MD, for providing clinical consultation on patient identification criteria and disease comorbidities, and Helen Woodroof, PhD, of Complete HealthVizion, for editorial assistance in the writing and revision of the draft manuscript on the basis of detailed discussion and feedback from all the authors; this assistance was funded by Allergan.

\section{REFERENCES}

1. Cash BD, Chey WD. Irritable bowel syndrome - an evidence-based approach to diagnosis. Aliment Pharmacol Ther. 2004;19(12):1235-45.

2. Longstreth GF, Thompson WG, Chey WD, Houghton LA, Mearin F, Spiller RC. Functional bowel disorders. Gastroenterology. 2006;130(5):1480-91.
3. Hulisz D. The burden of illness of irritable bowel syndrome: current challenges and hope for the future. J Manag Care Pharm. 2004;10(4):299-309. Available at: http://www.jmcp.org/doi/abs/10.18553/jmcp.2004.10.4.299.

4. Saito YA, Schoenfeld P, Locke GR. The epidemiology of irritable bowel syndrome in North America: a systematic review. Am J Gastroenterol. 2002;97(8):1910-15

5. Talley NJ, Boyce PM, Jones M. Predictors of health care seeking for irritable bowel syndrome: a population based study. Gut. 1997;41(3):394-98

6. Williams RE, Black CL, Kim HY, et al. Determinants of healthcareseeking behaviour among subjects with irritable bowel syndrome. Aliment Pharmacol Ther. 2006;23(11):1667-75.

7. Levy RL, Von Korff M, Whitehead WE, et al. Costs of care for irritable bowel syndrome patients in a health maintenance organization. Am J Gastroenterol. 2001;96(11):3122-29.

8. Doshi JA, Cai Q, Buono JL, et al. Economic burden of irritable bowel syndrome with constipation: a retrospective analysis of health care costs in a commercially insured population. J Manag Care Spec Pharm. 2014;20(4):382-90. Available at: http://www.jmcp.org/doi/abs/10.18553/jmcp.2014.20.4.382.

9. Chang L, Lembo A, Sultan S. American Gastroenterological Association Institute technical review on the pharmacological management of irritable bowel syndrome. Gastroenterology. 2014;147(5):1149-72.

10. Chey WD, Kurlander J, Eswaran S. Irritable bowel syndrome: a clinical review. JAMA. 2015;313(9):949-58.

11. Ford AC, Moayyedi P, Lacy BE et al. American College of Gastroenterology monograph on the management of irritable bowel syndrome and chronic idiopathic constipation. Am J Gastroenterol. 2014;109(Suppl 1):S2-S26.

12. Segel JE. Cost of illness studies-a primer. January 2006. Available at: http://www.amcp.org/WorkArea/DownloadAsset.aspx?id=12960. Accessed October 28, 2016.

13. Leong SA, Barghout V, Birnbaum HG, et al. The economic consequences of irritable bowel syndrome: a US employer perspective. Arch Intern Med. 2003;163(8):929-35

14. Mitra D, Davis KL, Baran RW. All-cause health care charges among managed care patients with constipation and comorbid irritable bowel syndrome. Postgrad Med. 2011;123(3):122-32.

15. Patel RP, Petitta A, Fogel R, Peterson E, Zarowitz BJ. The economic impact of irritable bowel syndrome in a managed care setting. J Clin Gastroenterol. 2002;35(1):14-20.

16. Elixhauser A, Steiner C, Harris DR, Coffey RM. Comorbidity measures for use with administrative data. Med Care. 1998;36(1):8-27.

17. Hawkins K, Wang S, Rupnow M. Direct cost burden among insured US employees with migraine. Headache. 2008;48(4):553-63.

18. Sullivan PW, Ghushchyan VH, Slejko JF, Belozeroff V, Globe DR, Lin SL. The burden of adult asthma in the United States: evidence from the Medical Expenditure Panel Survey. J Allergy Clin Immunol. 2011;127(2):363-69. 